\title{
BALANCE Y ANÁLISIS CRÍTICO DE LA INVESTIGACIÓN «FAMILIA: UNA JORNADA POR SU NATURALEZA, DERECHOS Y RESPONSABILIDADES» ${ }^{1}$
}

Virginia Aspe Armella
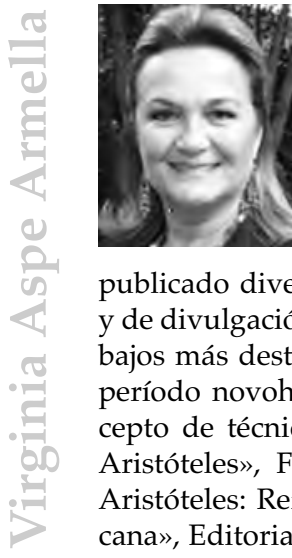

Profesora titular de Filosofía en México. Facultad de Filosofía de la Universidad Panamericana, sede México. Investigador Nivel 2. Miembro de la Academia Mexicana de Doctores en Ciencias Humanas y Sociales. Ha publicado diversos artículos en revistas especializadas y de divulgación, además de varios libros. Entre sus trabajos más destacados: «Las aporías fundamentales del período novohispano», CONACULTA (2002); «El concepto de técnica, arte y producción en la filosofía de Aristóteles», FCE (1993); «Perennidad y apertura de Aristóteles: Reflexiones poéticas y de incidencia mexicana», Editorial Cruz, O. (2004).

Correo electrónico: [virginiaaspe@yahoo.com.mx].

A partir del desarrollo del pensamiento moderno posterior a la Ilustración, hemos venido presenciando, cada vez más, el debilitamiento del valor familiar. En mi opinión, éste es un problema derivado de al menos tres principios familiares modernos:

1. La separación moderna de la consideración de lo humano a partir de la naturaleza.

2. La escisión entre lo público y lo privado, en la red de relaciones humanas.

3. Un error metódico al abordar las realidades éticas y políticas, desde las categorías de las ciencias fenoménicas.

Virgina Aspe et al., Familia: una jornada sobre su naturaleza, derechos y responsabilidades, UP-Porrúa, México, 2007, 344 p. 
El desarrollo de estos tres principios será el eje del trabajo que aquí presento, intercalando posibles modos de superarlos, según la acertada intervención de los distintos investigadores que realizaron conmigo el libro Familia: una jornada sobre su naturaleza, derechos y responsabilidades.

\section{SEPARACIÓN MODERNA DE LA CONSIDERACIÓN DE LO HUMANO A PARTIR DE LA NATURALEZA}

Diversos autores del libro abordan la problemática familiar separándose del error moderno de desvincular naturaleza y familia. El espléndido artículo de Miguel León Portilla, aunque no menciona principio alguno de la modernidad, pues su investigación es sobre la familia en el mundo prehispánico, establece las bondades de la interpretación náhuatl de la familia, cimentadas precisamente en la analogía que los antiguos mexicanos establecieron entre la familia y la naturaleza. Los textos del Códice Florentino citados por León Portilla afirman:

Oye bien, hijita mía, niñita mía, no es lugar de bienestar en la tierra, no hay alegría, no hay felicidad. Se dice que la tierra es lugar de alegría penosa, de alegría que punza.

Así andan diciendo los viejos: Para que no siempre andemos gimiendo, para que no estemos llenos de tristeza, el Señor Nuestro nos dio a los hombres la risa, el sueño, los alimentos, nuestra fuerza y nuestra robustez y el acto sexual, por el cual se hace siembra de gentes [...].

Pero, ahora, mi muchachita, escucha bien, mira con calma: he aquí a tu madre, tu señora, de su vientre, de su seno te desprendiste, brotaste. Como sale la hoja, así creciste, floreciste. Como si hubieras estado dormida y hubieras despertado $[\ldots]^{2}$.

En otro Códice, el Matritense de la Real Academia de la Historia, León Portilla extrae la significación de la familia náhuatl a partir de las metáforas con las que los antiguos mexicanos apuntan a lo más valioso, por encima de todo, en la realidad natural: 
El niñito: criatura, tortolita, pequeñito, tiernecito, bien alimentado [...]

Como un jade, una ajorca, turquesa divina, pluma de quetzal, cosa preciosa,

la más pequeñita, digna de ser cuidada $[\ldots]^{3}$.

Ello nos lleva a asemejar algunas tesis de León Portilla con textos de Carlos Llano Cifuentes, pues, aunque sean muy diversas, establecen una conexión entre familia y naturaleza. Para Llano, el compromiso familiar involucra un compromiso natural. La familia es una amistad no elegida, dada por la sangre, que procede de naturaleza. Sin embargo, considera que la familia no está exclusivamente en el terreno de las relaciones biológicas: el motivo de la vida común es la amistad, y la familia es el primer agente socializador de lo humano. Centrando su escrito en la conexión entre persona, Estado y ciudad, Llano menciona que más allá de todos los aspectos y modelos económicos y sociales, late siempre un conflicto antropológico. Para él, comprender la familia es conocer su naturaleza, por eso plantea, en otra de sus obras y de modo inverso que aquí, que el problema ecológico — el desorden de la naturaleza - es un problema ético, ¡del mundo de la libertad! Su discurso presenta una retroalimentación entre naturaleza y libertad muy importante.

Llano Cifuentes se separa expresamente de los principios modernos de la familia cuando distingue entre "comunidades personalmente vinculatorias» - la familia-, comunidades del Estado burocratizado moderno que son despersonalizantes, y comunidades del mercado de consumo que también tienen carácter impersonal. Señala que la familia es el primer agente socializador de lo humano y que es la única comunidad que establece redes que concilien la esfera de lo público y 
lo privado. La familia es capaz de establecer relaciones amistosas naturales: «relaciones hilemórfico-libres», como él las llama.

Contrario a autores modernos como Rousseau, Llano propone el reencuentro del hombre con la naturaleza para plenificar el Estado político y de la ciudad. Esta reconciliación es opuesta a lo que el francés sostiene en El Emilio donde afirma:

[...] todo es bueno al salir de las manos del autor de las cosas, todo degenera en las manos del hombre que obliga a un terreno a producir productos de otro, mezcla y confunde los climas, los elementos, las estaciones; el hombre mutila a su perro, a su caballo, a su esclavo; lo altera todo, lo trastorna todo, ama las deformidades y los monstruos ${ }^{4}$.

También contraria al abordaje roussoniano, encontramos la propuesta de Alberto Pacheco. Tomando la vuelta a la naturaleza como argumento de autoridad, Pacheco asume el principio de la heterogeneidad natural. Une vida humana y naturaleza al sentenciar que el matrimonio supone una relación natural entre distintos cuyo fruto son los hijos, y entre todos ellos se establece una comunidad indisoluble.

\section{LA ESCISIÓN ENTRE LO PÚBLICO Y LO PRIVADO EN LA RED DE RELACIONES HUMANAS}

Sigamos con el análisis del segundo error de los principios modernos en relación con la familia a través del estudio de nuestro libro Familia: una jornada...

Si para Carlos Llano sólo la familia es capaz de establece relaciones hilemórfico-libres, autores como Zagal, Sierra Madero, Martí Borbolla, Del Arenal, Jiménez Ottalengo, Villalobos y De la Mata abordan la problemática familiar recomendando el reencuentro de lo público y lo privado.

4 J. J. Rousseau, El Emilio o de la educación, Porrúa, México 1999, p. 1. 
Respecto a este enfoque, Marveya Villalobos propone repensar la familia. Sostiene que no hay una crisis de la esencia de la familia sino del modo en que se interpretar como modelo. Villalobos sigue las contribuciones de Mauricio Beuchot sobre hermenéutica analógica y nos recuerda que la familia es una institución natural flexible, abierta a distintos modelos culturales que mantienen una semejanza por analogía. Villalobos critica la estructura de las redes familiares modernas donde los valores democráticos han propiciado la pérdida de centralidad de los padres. Propone un modelo circular-familiar que converja al centro, aceptando que el modelo piramidal tradicional debe superarse, Villalobos insiste en no disolver la autoridad paterno-filial. Su modelo geométrico une lo público con lo privado, propone comunicación y participación vivida al modo democrático pero con autoridad en forma de alianza. Se trata de un dominio político familiar, no de un dominio despótico.

Otra unión entre familia y entorno es la que presentan Beatriz Quintanilla y Alejandra Tinajero. Quintanilla aborda patologías familiares. Después de un análisis acucioso de sus principales patologías, coloca la causa de los desequilibrios en la desintegración del yo y en la falta de conexión con el medio exterior, la vida pública. Tinajero sigue el mismo argumento pero respecto a la muerte en la familia. Con un análisis importante del flujo de las emociones en cuanto a dolor, duelo, pérdida y ansiedad, muestra cómo el cuidado y la confianza establecen puentes de cura y comunicación entre el mundo y la trascendencia.

El texto de Felipe Martí Borbolla es un análisis historiográfico-jurídico de la separación entre lo público y lo privado, y en esa misma tónica, Jaime del Arenal sostiene que la pérdida de los derechos comunitarios proviene de la Ilustración. Del Arenal señala que los códigos modernos se establecieron para salvar y proteger los derechos individuales. Dichos textos concebían las acciones en su conjunto como acciones individuales, no comunitarias. Ello trajo como consecuencia que la familia se interpretase y normase como un pacto individual, un procedimiento - técnica o artificio- ya no natural sino contractual. Este énfasis formalizante de relaciones solipsistas — cada vez más alejadas de la comunidad natural que caracterizaba a la familia-, produjo un modelo paterno-filial sin autoridad ni verticalidad alguna. En la familia moderna, de acuerdo a 
Del Arenal, el hijo no obedece al padre porque no existe ya una autoridad jerárquica, sino que los hijos «negocian» con los padres. Se trata de un nuevo modelo horizontal igualitarista del ámbito familiar que, al carecer de un vínculo real, de facto, funciona por mera regulación de técnicas y normas artificiales a la manera del Contrato social de Rousseau:

Hay que ir contra lo que uno mismo es para subsistir. Pero como uno es naturaleza no puede generar desde sí una nueva fuerza que le permita subsistir de manera distinta a lo que se es. Para lograrlo, el ser humano echa mano del modo propio que tiene, y así «puesto que los hombres no pueden generar nuevas fuerzas sino sólo unificar y dirigir las existentes, los hombres carecen de otro medio para conservarse que el dado, forman por agregación la suma de fuerzas que les permitan permanecer sobre la resistencia de los obstáculos» ${ }^{5}$.

Es así que, como decíamos, el punto de partida en la modernidad consistió en la separación del hombre y la naturaleza para después volver procedimental a la organización social. Se trató de renunciar a ciertos derechos en aras de un funcionamiento eficaz y seguro garantizando la tolerancia y las regulaciones formales. El hombre moderno concibe de modo trágico su estado natural y pretende reconstruir su ser por lo que no es, mediante la suma y potenciación de fuerzas de resistencia. La educación familiar se convierte entonces en modelos, métodos y técnicas eficaces. Este paradigma descuida el proceso de humanización de la comunidad optando por el desarrollo de estrategias poiéticas.

En este punto, la investigación de Héctor Zagal Arreguín cae como una brisa de aire fresco en el horizonte calcáreo. Zagal propone la continuidad entre ética y política porque ambos órdenes persiguen el mismo fin: la felicidad. Sugiere un nuevo paradigma para enfrentar lo público y lo privado, pues considera que el enfoque moderno entre derechos y obligaciones es contractualista, mientras que el lazo entre la esfera familiar y pública es la unidad del fin que persiguen: ambos establecen la excelencia de la función propia en el bien común. Para Zagal, la superación del 
contractualismo moderno en la familia se justifica porque en ella existe un cierto cariz de benevolencia y gratuidad, cuyo sentido indica que la familia no se rige en sus relaciones por la justicia. La desigualdad es la marca de las relaciones familiares porque en la familia cada uno tiene diversas funciones según sus distintas capacidades y aptitudes.

El artículo de Adame Goddard es un ejemplo en la conexión que Zagal recomienda. Parte de elementos naturales y de sujetos morales para escudriñar la amistad en la familia y su proyección sociopolítica desde la naturaleza y justicia del matrimonio. En el texto de Felipe de la Mata observamos cómo se aborda frontalmente que la noción de matrimonio se ha ampliado para incluir al concubinato como una especie de unión conyugal en la legislación del Distrito Federal.

Congruente con el postulado de Adame Goddard, Regina Jiménez Ottalengo sostiene que la familia es un sujeto social que debe defenderse porque es la generadora del capital social primigenio. Con un abordaje crítico sumamente novedoso, Jiménez Ottalengo enfrenta el problema familiar del consumismo recordando la necesidad del trabajo: hay que restituir a la familia su papel de actividad productora de una sociedad. La familia es una comunidad, inscrita en el marco de las dimensiones económicas y sociales. Esta propuesta aterriza las relaciones entre éthos y polis que Zagal venía recomendando. En mi opinión, los escritos de Carlos Llano Cifuentes, Regina Jiménez Ottalengo y José Luis Ortiz, enfrentan dos de las tres problemáticas más acuciantes de la familia, hoy; me refiero a las relaciones entre familia/economía, familia/medios, y familia/violencia/adicciones. Martí Borbolla apunta a una solución del problema restituyendo el sentido de comunidad. Para él, la solución radica en plantear la familia desde los derechos civiles, sociales y culturales.

José Luis Ortiz enfrenta el tema de la familia y los medios con una propuesta constructiva, al igual que Jiménez Ottalengo. Para Ortiz, hay que hacer virtuoso el problema mediático, aprovechando los contenidos que se trasmiten. Establece un modo de conciliar lo público de los medios y lo privado en la televisión familiar a través de la virtud. Ortiz propone aprender a usar los medios, educar a la familia para recibir sus mensajes, 
construir lazos virtuosos entre la vida privada y la comunicación de masas mediante rectos usos y el aprovechamiento de la información.

Mencioné anteriormente que los tres problemas más acuciantes de la familia actual son la economía, los medios, y la violencia y las adicciones. Estudios recientes demuestran una estrecha conexión entre medios de comunicación, violencia familiar y adicciones. No me detendré aquí a analizar a fondo cada uno de estos problemas, pero sí quiero recordar, a raíz de estas Jornadas de familia, que los medios son hoy la principal influencia del entorno familiar. Frente a la vieja enunciación griega de que el conocimiento es tabula rasa y que su punto de partida es la realidad natural, al presente debemos preguntarnos si el punto de partida del conocimiento sigue siendo el mismo para un griego del siglo IV a.C. y para un niño citadino mexicano del siglo XXI.

¿Qué es lo primero que se conoce en el entorno familiar? En Atenas, seguramente eran los elementos y las realidades naturales. Cuenta un viejo texto griego que Calias, desde pequeño, observaba el mar junto al árbol de olivos de su casa y así comenzó a preguntarse por la realidad. Pero los nuevos relatos sobre niños mexicanos del Distrito Federal cuentan que se debe prevenir que vean un video japonés parecido al de «Pokemon» porque les dan ataques epilépticos. Lo primero que hoy conocen no es la naturaleza, sino un mundo mediatizado y con videos cibernéticos. Este paradigma nos enfrenta a nuevos retos en el marco educativo familiar. Así como en el siglo pasado, en la década de los 20, durante el México cristero, quizás el argumento de autoridad se enunciaba con un: «Me lo dijo el Padre de la Peza», hoy el argumento familiar puede ser un juego de video, la información de un programa del National Geographic o la solución que se facilitó a un problema marital en un talk-show.

En esta posmodernidad nos encontramos frente a nuevos paradigmas familiares que nos llegaron como cae una pedrada en la cabeza por detrás: sin percatarnos. Para colmo, enfrentamos la nueva realidad medial del conocimiento y la educación familiar con planteamientos débiles, heredados de la modernidad. Sin afán de ser macabra ni deprimente, acepto que los planteamientos consecuencialistas éticos en la familia hoy son el pan de cada día: damos más importancia al éxito, a las calificaciones, al triunfo 
económico y al bien portarse, que a la valentía, a las convicciones profundas o al reto de que los hijos se enfrenten a nuevos modelos de modo creativo o hagan denuncias incómodas pero comprometidas. No sabemos qué hacer con la idea de un matrimonio débil y cuando alguien aguanta penurias matrimoniales pensamos o que es fundamentalista o que lo hace por temores religiosos y le falta valor. Asistimos a la legalización de relaciones de homosexuales, con posibilidad de adopciones, y de relaciones de parejas sin compromiso. Observamos la falta de roles heterogéneos y una tendencia a desequilibrios sociales importantes. El estudio de Rodrigo Guerra sobre este problema nos descubre cifras interesantísimas del desequilibrio en los modelos familiares mexicanos para los próximos años.

Pero no debemos magnificar estas situaciones. Todas las épocas han tenido problemas familiares acuciantes y ellos han sido el acicate para nuevas investigaciones y avances hacia el interior del núcleo familiar. En concreto, hoy, frente a estos problemas, se han apuntado soluciones y propuestas francamente alentadoras. Marveya Villalobos señala este punto cuando escribe sobre repensar los modelos familiares en la diversidad cultural; y el estudio inmejorable de Luis Xavier López-Farjeat sobre la familia musulmana enfrenta con valentía otros modelos familiares y sus contribuciones actuales. Eso mismo hace el estudio de León Portilla trayendo al aquí, las contribuciones de la familia prehispánica. En ellos dos y en Héctor Zagal advertimos cómo el intelectual posmoderno se abre desprejuiciadamente a otras culturas para repensar la familia de un modo más abierto.

Alberto Pacheco contribuye también a este esfuerzo, poniéndonos al día en lo relativo al matrimonio católico desde el derecho canónico.

En otra línea de investigación, pero con esta misma actitud propositiva y abierta, el texto de Dora Sierra Madero informa sobre contribuciones jurídicas para la familia actual. Se trata del análisis profundo sobre las reformas jurídicas para encauzar a la familia. Lo mismo que Dora Sierra, actualmente muchos especialistas internacionales proponen nuevas soluciones para el modelo tradicional familiar. Algunos hablan de proteger pública y legalmente la concepción como unión de hombre y mujer. 
Otros, proponen mejorar el sistema fiscal de las familias como incentivo para preservar su permanencia y valores. Algunos intentan proteger los derechos del niño por nacer mediante procreación artificial, pues se encuentran desprotegidos al ser considerados meros objetos de consumo.

Los estudios más especializados intentan romper el círculo vicioso que afirma que la familia genera crisis sociales y las crisis sociales engendran rupturas familiares.

En lo que sí hay consenso actualmente es en la necesidad de integrar lo privado y lo público, estableciendo lazos entre la vida ética y la civil. También, hoy es clara la necesidad de abrir más el cerco privado del ámbito familiar en lo referente a problemas como violencia intrafamiliar, adicciones y abusos sexuales. Estas tres problemáticas familiares deben ser analizadas como hechos sociales producidos por causas múltiples, siendo las más influyentes:

1. Ausencia de sentido de la vida por la falta de integración de un yo con personalidad confiada y estable, fruto de un entorno familiar que proporciona atención, cariño y formación humana. La violencia y disfunción familiar inician con esta ruptura.

2. Ausencia de una conexión entre la esfera familiar y la pública. Las adicciones tienen su origen en esta falta de conexión, aunada a patrones aprendidos a través de los medios de comunicación.

3. Falta de recto uso frente a los medios masivos de comunicación.

4. Ruptura del modelo tradicional familiar por efectos de la globalización, migraciones, pérdida de roles heterogéneos, consumismo y economía liberal desordenada.

Presento aquí las recomendaciones del último seminario internacional sobre violencia familiar y adicciones. El hallazgo que presenta este grupo interdisciplinario en el marco de políticas públicas internacionales para una educación preventiva familiar, afirma:

Desde finales del siglo pasado, se ha venido planteando una revolución paradigmática orientada hacia la complejidad, en los planos de las ciencias físicas, del conocimiento del hombre y de la política. El planteamiento va 
encaminado a sustituir la idea tradicional de objeto: cerrada, monótona y uniforme, por la de sistema: en la cual interactúan dialógicamente orden y desorden.

Como vemos, las propuestas de los autores que conforman la presente obra, están en sintonía con esos grupos de vanguardia.

Cierro mi intervención, desarrollando el último punto de los tres que señalé al inicio.

\section{UN ERROR METÓDICO EN LA MODERNIDAD QUE PRETENDIÓ ABORDAR LAS REALIDADES ÉTICAS Y POLÍTICAS CON LAS MISMAS CATEGORÍAS QUE LAS CIENCIAS FENOMÉNICAS}

El libro abre precisamente con esta precisión metódica: en mi artículo, presento un análisis de la teoría de la ciencia aristotélica para probar que el mismo Aristóteles — padre del silogismo demostrativo, autor de un modelo científico causal, universal y necesario- reconoce la diversidad de accesos metódicos para distintos géneros de investigación. Aristóteles privilegia el silogismo demostrativo necesario y causal especialmente en la matemática. Pero en Analíticos posteriores habla ya de modelos fenoménicos distintos en su término medio al formal, porque en las ciencias de la naturaleza se involucra la materia intrínsecamente a la demostración. Propone ejemplos como el de los eclipses, la salud y el desarrollo de las plantas y, aunque no se refiere a una diversa forma de silogismo para los razonamientos éticos y políticos, sí adelanta la conversión -en griego, anagogé- que otros modelos demostrativos tendrían que hacer para adaptarse al género que tratan.

Para Aristóteles, las realidades ético-políticas pueden abordarse científicamente en analogía con los procesos naturales. Conviene mencionar aquí que Aristóteles habla del método que debe utilizarse en las ciencias subalternas. Ello prueba que no emplea siempre el mismo modo de demostración en distintas realidades. En la Ética Nicomaquea, señala: 
[...] es propio del hombre instruido buscar la exactitud en cada materia en la medida en que lo admite la naturaleza del asunto; evidentemente, tan absurdo sería aceptar que un matemático empleara la persuasión, como exigir de un retórico demostraciones ${ }^{6}$.

Y, aunque en la Política sitúa a la familia por el lado de las relaciones por necesidad - no de libertad, como en la vida ciudadana- explica que el fin de la vida doméstica es el bien de la comunidad. Con esto, Aristóteles enlaza necesidad y libertad en una peculiar forma de comunidad previa a la autarquía de la polis pero cuyo mismo fin establece una continuidad transversal en los valores.

En Adame Goddard tenemos un ejemplo paradigmático del abordaje transversal. Acomete la realidad jurídica familiar desde la Nicomaquea de Aristóteles para trascender el éthos familiar y anunciarlo a la luz de su función política.

El artículo de Ana Tere López de Llergo se pregunta por esta metodología, tanto para el currículo de las asignaturas como para la educación familiar, porque en el ámbito de la formación de valores y actitudes, sólo una metodología transversal puede penetrar y cumplir las expectativas de un género-sujeto heterogéneo, multifactorial como es la familia. Al mismo tiempo, la investigación familiar ha de tener un primer analogado, pero ello debe buscarse desde un centro de antropología social que permita abordar cuestiones familiares con proporcionalidad. Mi análisis de la metodología científica para la investigación familiar no busca un saber científico matemático, sino la estabilidad razonable que permite el saber prudencial para llegar a verdades generales estables.

Esta vuelta al viejo modelo griego que veía la realidad humana de modo heterogéneo, supone un cambio en el paradigma metódico moderno excesivamente centrado en la objetividad y, en consecuencia, aislacionista de variables alternativas. La transversalidad reconoce distintos ámbitos, entiende que la formación ética es también civil, que en ella se

Aristóteles, Ética Nicomaquea, libro I, 1094b12-1095a11. 
involucran la cultura, las emociones, las diversas creencias, los procesos psicoafectivos, la narrativa, el modo de representación, los imaginarios, la creatividad y el ingenio. Educar, formar, no es transmitir códigos morales ni meras ideologías por tradiciones o intereses de tipo religioso.

Es indispensable repensar el método adecuado para plantear la problemática actual familiar más allá del patrón sociológico moderno. Nuevos grupos de especialistas en familia recomiendan una metodología transversal para la formación ética y cívica, sin darse cuenta de que su «gran hallazgo» se gestaba ya en la paideia griega y pasaba al imaginario del alto medioevo.

Es indudable que el enfoque heterogéneo de la realidad humana implica una metodología transversal que supere planteamientos univocistas. En López de Llergo y Aspe se patentiza la necesidad de escudriñar el nuevo modo de plantear la familia para evitar dogmatismos y univocidades y, así, abrirnos a los distintos modelos familiares sin perder su esencia. López-Farjeat, León Portilla, Pacheco y Zagal enfrentan por eso viejos modelos culturales y el modo como ellos se representaban las relaciones entre familia y entorno a la luz de la tradición. De la Mata, Martí Borbolla, Del Arenal, Adame Goddard y Sierra Madero, lo hacen desde el punto de vista jurídico. Ortiz, Quintanilla, Villalobos, Jiménez Ottalengo, Llano Cifuentes y Tinajero rastrean la conexión entre la familia como comunidad íntima y el entorno como realidad que la trasciende. Pienso que ésta ha sido la unidad de un proyecto de investigación fresco, espontáneo, porque ha pensado los problemas sin ideología alguna, sin línea en la investigación y que, sin embargo, presenta continuidades. 


\section{BIBLIOGRAFÍA}

- ASPE, Virgina et al., Familia: una jornada sobre su naturaleza, derechos y responsabilidades, UP-Porrúa, México, 2007.

- Códice Florentino, edición facsimilar, 3 vols., México, 1979.

- Códice Matritense de la Real Academia de la Historia (textos en náhuatl de los indígenas informantes de Sahagún), edición facsimilar de Del Paso y Troncoso, vol. VIII, Madrid, 1907.

- ARISTÓTELES, Ética Nicomaquea, Gredos, Madrid. 1982.

- ARISTÓTELES, Analíticos posteriores, Aguilar, Madrid, 1982.

- ARISTÓTELES, Política, UNAM. México. 2000. 